\section{Prevalence of Congenital Myopathies in a Representative Pediatric United States Population}

\author{
Kimberly Amburgey, MS, ${ }^{1}$ Nancy McNamara, MD, ${ }^{1}$ \\ Lindsey R. Bennett, MS, CGC, ${ }^{2}$ \\ M. Eileen McCormick, DO, ${ }^{3}$ Gyula Acsadi, MD, PhD, ${ }^{4}$ \\ and James J. Dowling, MD, PhD ${ }^{1}$
}

The prevalence of congenital myopathies in the United States has not been examined. To address this, we determined the point prevalence of congenital myopathies in a well-defined pediatric population from Southeastern Michigan. The overall point prevalence was $1: 26,000$. Mutations in RYR1 were the most common cause of congenital myopathies at 1:90,000. Our data broadly agrees with estimates from previous European studies and provides the first estimate of the prevalence of congenital myopathies in the United States.

ANN NEUROL 2011;70:662-665

C ongenital myopathies are a heterogeneous group of neuromuscular disorders that typically present in childhood. ${ }^{1}$ They are largely defined by clinical presentation and characteristic features on biopsy. While prevalence data from multiple different countries exists for Duchenne muscular dystrophy and spinal muscular atrophy, ${ }^{2}$ much less is known about the epidemiology of congenital myopathies. Historically, this group of conditions is considered to be quite rare, though no single study has exclusively and systematically examined them.

Three previous studies provided estimates of the prevalence of these disorders. One study examined a cohort from Northern Ireland and estimated the prevalence of congenital myopathies (adult and pediatric) as $1: 28,600 .^{3}$ A retrospective epidemiologic study of pediatric neuromuscular cases performed for western Sweden determined the prevalence of congenital myopathies as $1: 22,480 .{ }^{4}$ Additionally, they found that the most prevalent specific subtype was nemaline myopathy $(1: 179,000)$, though unspecified congenital myopathies were significantly more common $(1: 27,000)$. Most recently, the prevalence of neuromuscular disorders was examined in a cohort from northern England. ${ }^{5}$ In this study, the prevalence of congenital myopathies (adult and pediatric) was $1: 135,000$, with central core disease the most prevalent at 1:249,000.

Currently, no estimates exist concerning the prevalence of congenital myopathies in the United States. Barriers to defining such data are the following: (1) the large size of the U.S. population; (2) the lack of centralization of neuromuscular centers; and (3) the lack of accessible records for epidemiologic studies. One approach for overcoming these problems is to examine a well-defined subset of the population. In the current study, we have taken such an approach by performing an epidemiologic analysis of the prevalence of congenital myopathies in the pediatric population of southeastern Michigan. This population is large (approximately 1.2 million aged 0-17 years), has demographics similar to the overall U.S. population, and is serviced by a defined number of pediatric neuromuscular clinics. ${ }^{6}$

We present the prevalence data from our epidemiologic study. We found that the overall point prevalence in 2010 of confirmed pediatric cases of congenital myopathies is $1: 26,000$. The largest subgroup was the unspecified group (prevalence $=1: 55,000$ ), whereas the most common specific histopathologic subtype was the core myopathies $(1: 80,000)$. In all, our study provides the first estimate of the frequency of congenital myopathies in a pediatric population in the United States and provides a comparison between this population and those examined in previous studies.

\section{Patients and Methods}

\section{Catchment Area}

The population of the state of Michigan is divided into welldefined geopolitical regions. The largest region in the state is the Ann Arbor-Detroit-Flint area in southeastern Michigan (Fig 1). According to the last systematic examination of this area by the U.S. Census bureau in $2007,{ }^{6}$ the population of individuals under the age of 18 years is $1,211,100$. The demographics for this group are $75 \%$ white, $22 \%$ black or AfricanAmerican, and 3\% Latino. The overall demographics for the United States population are $80 \%$ white, $13 \%$ black or AfricanAmerican, and 16\% Latino. ${ }^{6}$

\section{Ascertainment}

We hypothesized that all, or nearly all, pediatric patients with congenital myopathies receive clinical care in a Muscular Dystrophy Association (MDA) sponsored clinic. Currently, there are 3 MDA clinics in the Ann Arbor-Detroit-Flint area that provide

From the ${ }^{1}$ Department of Pediatrics, University of Michigan, Ann Arbor, $\mathrm{Ml}^{2}{ }^{2}$ Department of Neurology, Children's Hospital of Michigan, Detroit, $\mathrm{Ml} ;{ }^{3}$ Michigan Institute for Neurological Disorders, Farmington Hills, Ml; and ${ }^{4}$ Department of Pediatrics, Connecticut Children's Medical Center, Hartford, CT.

Address correspondence to Dr Dowling, 5019 BSRB, 109 Zina Pitcher Place, Ann Arbor, MI 48109-2200. E-mail: jamedowl@med.umich.edu

Received Mar 4, 2011, and in revised form May 3, 2011. Accepted for publication Jun 3, 2011.

View this article online at wileyonlinelibrary.com. DOI: 10.1002/ana. 22510 




FIGURE 1: The lower peninsula of the state of Michigan (adapted from the Michigan Business Directory). The Ann Arbor-Flint-Detroit catchment area is outlined by a black line. The estimated pediatric population in this area is $1,200,000$.

clinical services for children. We collected clinical and diagnostic data on patients from these clinics using a retrospective chart review. Duplicate cases were excluded by using individual identifying information. Chart reviews were performed over a 3-month period between September 2010 and December 2010.

\section{Inclusion Criteria}

Inclusion criteria for the study were as follows: (1) age under 18 years (as of December 2010), (2) alive in 2010, (3) residence in Michigan, (4) evaluation in 1 of the 3 MDA clinics between January 2005 and December 2010, and (5) a confirmed diagnosis of congenital myopathy. The diagnosis of congenital myopathy was based on a consistent clinical history and at least 1 additional supporting study (biopsy, genetic testing, or first-degree relative). The subtypes included in this study were centronuclear myopathy, central core disease, multiminicore disease, nemaline myopathy, and congenital fiber type disproportion. Nonspecific biopsies were defined as those containing myopathic features without evidence of a defined histopathological subtype.

\section{Exclusion Criteria}

Cases with a clinical diagnosis of congenital myopathy without supporting confirmatory data were not included in the prevalence totals. In addition, patients with a concomitant diagnosis (such as Down syndrome, other neuromuscular disease, etc.) were also excluded from the study.

\section{Institutional Review Boards}

This study was reviewed by the Institutional Review Boards (IRBs) at the University of Michigan and at Wayne State Uni- versity. IRB approval numbers were: HUM00030796 and HIC081009MP2E.

\section{Results}

\section{Overall Point Prevalence}

A total of 62 patients were identified with a clinical diagnosis of congenital myopathy. Of these patients, 46 had diagnostic testing (41) or family history (5) consistent with a congenital myopathy and thus were included in the point prevalence calculation. Using a population estimate for southeastern Michigan of 1,211,100, the overall point prevalence for pediatric congenital myopathies in this region is $1: 26,000$ (Fig 2).

\section{Subtype Prevalence}

HISTOPATHOLOGIC SUBTYPE. Congenital myopathies are most commonly defined by biopsy pattern. In our cohort, the most common specific histologic subtypes are minicore myopathy and central core disease, representing 8 of 44 and 7 of 44 biopsies, respectively. The largest proportion of cases $(22 / 44)$ is represented by those with a nonspecific biopsy pattern. Of these cases, 10 biopsies showed type I fiber predominance and 15 showed fiber atrophy/hypertrophy. The prevalence data for all subtypes is presented in the Table.

GENETIC SUBTYPE. A causative genetic mutation was identified in 16 of 46 cases. Thirteen patients had RYRI mutations. The prevalence of myopathies due to mutations in RYR1 (including 2 cases in which the sibling or parent had a positive gene test) is $1: 90,000$. Other causes of congenital myopathies and other related disorders were ruled out in several patients through genetic testing



FIGURE 2: Breakdown by percentage of each congenital myopathy subtype based on biopsy findings. $C C D=$ central core disease; CFTD = congenital fiber-type disproportion; $\mathrm{CNM}=$ centronuclear myopathy; $\mathrm{MmD}=$ multiminicore disease. 


\begin{tabular}{|c|c|c|c|}
\hline \multicolumn{4}{|c|}{ TABLE: Prevalence Data for all Subtypes } \\
\hline & Total & $\begin{array}{l}\text { Genetically } \\
\text { Confirmed }\end{array}$ & Prevalence \\
\hline $\begin{array}{l}\text { Clinical } \\
\text { diagnosis only }\end{array}$ & 16 & N/A & $1: 75,000$ \\
\hline $\begin{array}{l}\text { Nonspecific } \\
\text { biopsy }\end{array}$ & 22 & 5 & $1: 55,000$ \\
\hline CNM & 3 & 1 & $1: 400,000$ \\
\hline \multicolumn{4}{|l|}{$\begin{array}{l}\text { Core } \\
\text { myopathies }\end{array}$} \\
\hline CCD & 7 & 5 & $1: 170,000$ \\
\hline $\mathrm{MmD}$ & 8 & 3 & $1: 150,000$ \\
\hline Nemaline & 1 & 1 & $1: 1,210,000$ \\
\hline CFTD & 5 & 1 & $1: 240,000$ \\
\hline Total & 62 & 16 & $1: 26,000$ \\
\hline
\end{tabular}

including: myotonic dystrophy (13/46), RYR1 (4/46), SEPN1 (4/46), LMNA (2/46), ACTA1 (1/46), TPM2 (1/ 46), TPM3 (1/46), DNM2 (1/46), MTM1 (1/46), COL6A1-3 (3/46), SMN1 (4/46), PYGM (1/46), PFKM (1/46), deletion of 4q35 (FSHD) (2/46), and enzymatic testing for Pompe disease (1/46).

\section{Dystrophinopathy Prevalence}

As an internal control, we collected the number of dystrophinopathy patients. We identified 169 male patients with genetically confirmed dystrophinopathy with a prevalence of 1:7,000.

\section{Discussion}

We present for the first time the prevalence of congenital myopathies in a representative pediatric United States population. By examining a defined population from Southeastern Michigan, we determined a prevalence of $1: 26,000$. We predict that this number accurately reflects the overall pediatric prevalence of congenital myopathies in the United States. This is for 3 reasons: (1) the demographics of our cohort is similar to that of the general U.S. population, (2) our ascertainment "control" cohort (ie, dystrophinopathy patients) revealed a number (1:3,500 boys) in keeping with known calculations ${ }^{7}$ and thus indicative of complete or near complete ascertainment in our defined population, and (3) our prevalence number shows excellent agreement with those calculated in previous European studies (1:22,000 in Sweden and
1:28,000 in Northern Ireland). The exception was the recent study from Northern England, which determined prevalence as $1: 135,000$. This discrepancy is likely the result of the fact that this estimate did not include cases of nonspecific congenital myopathies.

Of note, given that our study was confined to a pediatric population, our estimate likely is an underrepresentation of the overall prevalence of congenital myopathies in the United States. It does not account for cases with adult onset or cases with mild pediatric onset that are not diagnosed until adulthood. However, as the majority of patients with congenital myopathies have onset and diagnosis in childhood, we hypothesize that our number represents a good estimate of the overall burden of these disorders. The fact that our prevalence number is very similar to the previous European studies (which included all ages) supports this conclusion.

Two additional significant findings are that core myopathies are the most common specific histopathologic subtype, and that congenital myopathies due to mutations in $R Y R 1$ are by the far the most prevalent genetic subtype in our population. This is in keeping with the recent explosion in the medical literature of reports of RYR1 mutations in essentially all histologic subtypes of congenital myopathies. ${ }^{8-10}$ This finding is also consistent with the data from northern England, but is at odds with the study from Sweden. In the Swedish cohort, nemaline myopathy was the most common subtype. This may reflect ethnic and geographic variability in terms of subtype distribution, as we uncovered only a single case of nemaline in our population.

The largest subgroup within our cohort was the "nonspecific" group. Patients in this group had a suggestive clinical history and a biopsy with nonspecific myopathic features. This was also the largest group in the Swedish study. It is unclear whether this group represents cases that will ultimately have a specific diagnosis, will continue to remain undefined, or will instead be recognized as having a different muscle condition that does not fall in the congenital myopathy spectrum. The fact that so many cases are undefined underscores the important point that muscle biopsy is often not sufficiently diagnostic. Additional and potentially novel modalities are thus needed in many cases to establish the ultimate diagnosis.

In all, we have established for the first time the prevalence of congenital myopathies in a representative U.S. subpopulation. We have also identified RYR1 mutations as the commonest genetic cause of congenital myopathies. We thus recommend a high degree of suspicion for $R Y R 1$ mutations in all genetically undefined cases of congenital myopathy. 


\section{Acknowledgments}

This research was supported by a grant from the NIH (UL1RR024986).

\section{Potential Conflict of Interest}

J.J.D., K.A., and N.M. have received a grant from NIH (UL1RR024986).

\section{References}

1. Sewry CA, Jimenez-Mallebrera C, Muntoni F. Congenital myopathies. Curr Opin Neurol 2008;21:569-575.

2. Emery AE. Population frequencies of inherited neuromuscular diseases-a world survey. Neuromuscul Disord 1991;1:19-29.

3. Hughes MI, Hicks EM, Nevin NC, Patterson VH. The prevalence of inherited neuromuscular disease in Northern Ireland. Neuromuscul Disord 1996;6:69-73.
4. Darin N, Tulinius M. Neuromuscular disorders in childhood: a descriptive epidemiological study from western Sweden. Neuromuscul Disord 2000;10:1-9.

5. Norwood FL, Harling C, Chinnery PF, et al., Prevalence of genetic muscle disease in Northern England: in-depth analysis of a muscle clinic population. Brain 2009;132(Pt 11):3175-3186.

6. US Census Bureau 2007

7. CDC 2007.

8. Clarke NF, Waddell LB, Cooper ST, et al., Recessive mutations in RYR1 are a common cause of congenital fiber type disproportion. Hum Mutat 31:E1544-E1550.

9. Wilmshurst JM, Lillis $\mathrm{S}$, Zhou H, et al., RYR1 mutations are a common cause of congenital myopathies with central nuclei. Ann Neurol 2010;68:717-726

10. Zhou H, Jungbluth $H$, Sewry CA, et al., Molecular mechanisms and phenotypic variation in RYR1-related congenital myopathies. Brain 2007;130(Pt 8):2024-2036 